\title{
Thymic Epithelial Cell Capable of Differentiating Towards Both Cortical and Medullary Cell Type
}

National Cancer Institute

\section{Source}

National Cancer Institute. Thymic Epithelial Cell Capable of Differentiating Towards Both

Cortical and Medullary Cell Type. NCI Thesaurus. Code C45704.

A reticular epithelial cell generated in the thymus that, in optimal conditions, can become either a cortex type of thymus cell that mediates positive selection or a medullary type of thymus cell that mediates negative selection of developing thymocytes. 